\title{
Research on Simulation of Electric Energy Substitution Policy
}

\author{
Peng $\mathrm{Wu}^{1}$, Yu Zhang ${ }^{1, *}$, Baoguo Shan ${ }^{1}$, Houqi Dong ${ }^{2}$, Mo Shi ${ }^{2}$ and Peng Zheng ${ }^{3}$ \\ ${ }^{1}$ State grid energy research institute Co., Ltd, Beijing, China \\ ${ }^{2}$ North China electricity power university, Beijing, China \\ ${ }^{3}$ State Grid Fujian Electric Power Co., Ltd., Fuzhou, China \\ ${ }^{*}$ Corresponding author
}

\begin{abstract}
Proper incentive policy guidance is the key to the successful implementation of electric energy substitution strategy. At present, there is a lack of evaluation method for the effect of electric energy substitution policy. Quantitative policy simulation analysis method is needed to provide support for scientific decision-making. In this paper, the electric energy substitution policy is regarded as an intervening event in the process of electric energy substitution, and an intervention analysis model of energy substitution time series is established. Through the intervention analysis on the growth rule of electric energy substitution in the policy implementation, the structural change of the growth trend of energy substitution can be quantified.
\end{abstract}

Keywords-electric energy substitution; policy simulation; policy intervention analysis model

\section{INTRODUCTION}

Perfect incentive policy guidance is the key to the successful implementation of electric energy substitution strategy. As our country's current energy price formation mechanism is imperfect, does not contain the environmental cost, resulting in price distortions ${ }^{[1]}$. Electricity does not have a comparative advantage comparing with coal, natural gas and other energy in the current price system, and it is difficult to change the way they use energy without policy incentive ${ }^{[2]}$. Therefore, a perfect incentive policy which guides the society to take the initiative to choose electric energy and form a way to eliminate high pollution and low efficiency is the key to the successful implementation of electric energy substitution strategy ${ }^{[3-4]}$. The current policy of electric energy substitution in our country mostly stays at the level of guidance and appeal, lacks actual measures to land on the ground. It's necessary for the quantitative policy simulation analysis method to provide support for scientific decision-making.

In the field of electric energy substitution, the index of electric energy substitution will also fluctuate under the influence of events, such as policy direction, industrial structure, technological progress and so on ${ }^{[5]}$. These indicators tend to be short-lived, and some policies may last only one year. The impact disappears when the policy changes after one year. However, some events have a long-term and sustained impact on electric energy substitution ${ }^{[6]}$, for example, the planning of new power industries, the long-term planning of urban construction, etc. These factors will lead to a sudden change in the growth trend of electric energy substitution, and continue to make a new growth trend of electric energy substitution.
In order to evaluate the influence of these factors on the normal time series, the intervention analysis model was introduced to evaluate the effect of emergency events on the variables ${ }^{[7]}$. In this paper, the power substitution policy is regarded as an intervening event in the process of electric energy substitution, and an intervention analysis model of energy substitution time series is established. Through the intervention analysis on the growth rule of electric energy substitution in the policy implementation, the structural change of the growth trend of energy substitution can be quantified. It can provide a lot of valuable information for the Energy Department, and help them to make better decisions on power grid planning, operation optimization and improving operation service efficiency.

\section{ClASSIFICATION AND MODELING OF ELECTRIC ENERGY SUBSTITUTE POLICY}

When the time series is a stationary non-white noise sequence, a linear model is established to fit the time series, and useful information is extracted from the time series. Autoregressive Moving Average Model (ARMA) is the most commonly used stationary time series fitting model. Basic steps for modeling analysis of power substitution sequences:

a) According to the scatter graph, autocorrelation function and partial autocorrelation function graph of energy substitution time series, the variance, trend and seasonal variation regularity of ADF unit root are examined, and the smoothness of time series is identified. Generally speaking, the time series of power substitution is non-stationary time series.

b) The Non-stationary time series data is processed smoothly. If the data sequence is non-stationary and there is a certain growth or descent trend, the data need to be treated with differential processing. The treatment means include difference and seasonal difference, etc.

c) The corresponding time series model is established based on the identified characteristics. The identification parameters of the model include difference order $d$, seasonal difference order $D$ and model order $p, q, P, Q$, which are mainly based on autocorrelation function and partial autocorrelation function. After the sequence is smoothed, if the partial autocorrelation function is truncated and the autocorrelation function is trailing, the sequence is suitable for the $A R$ model; if the partial autocorrelation function is trailing and the autocorrelation function is truncated, the sequence is suitable for the $M A$ model; 
if the partial autocorrelation function and autocorrelation function are trailing, the sequence is suitable for the $A R M A$ model.

d) The parameters in the model are estimated to test whether it is statistically significant. When the order of the model is determined, the parameters of each part of the model are estimated by using the effective fitting method, such as least squares estimation and maximum likelihood estimation.

e) Hypothesis test judges (diagnoses) whether the residual sequence of power substitution is white noise sequence.

f) The tested model is used to predict and analysis the goal.

Policy impact intervention analysis assuming that intervention exerts an influence on the process by changing the mean function or trend of the time series. According to the theory of time series intervention model (TTSIM) ${ }^{[8]}$, considering the impact of a particular policy on intervention, after appropriate transformation, the general model of time series $\left\{Y_{t}\right\}$ can be expressed as:

$$
Y_{t}=\left\{\begin{array}{lr}
N_{t}, & t<T \\
m_{t}+N_{t}, & t \geq T
\end{array}\right.
$$

Where $m_{t}$ is the impact of external intervention; $N_{t}$ is ARIMA model, including others impacts. $N_{t}$ is baseline time data not affected by policy intervention. The intervention analysis model is applied to the influence of power substitution policy on power substitution index. $m_{t}$ is the influence of electric energy substitution policy on energy substitution index, and $N_{t}$ is an indicator of electrical energy substitution that is not affected by the policy of electrical energy substitution. Suppose the policy is carried out at $T$ moment, that is, the time series of electrical energy substitution index is interfered at $T$ moment. Before $T$ moment, $\left\{Y_{t}\right\}$ is electric energy substitution time series without interference, which can identify electric energy substitution models before policy implementation.

\section{A. Policy Intervention Variables}

The basic variable of the power Substitution policy intervention analysis model is the power substitution policy intervention variable. Assuming the policy intervention variable as a virtual variable with a value of 0 or 1 , the intervention variable was 1 when the intervention took place and 0 when the intervention did not take place. The policy intervention variables are divided into two forms according to TTSIM: One is to indicate that the intervention has continued to have an impact after the $T$-moment, for example, long-term planning of energy substitution policies, an intervention variable that can be expressed as a ladder function:

$$
S_{t}^{(T)}=\left\{\begin{array}{l}
0, t<T \\
1, t \geq T
\end{array}\right.
$$

The other is that the intervention occurs at $T$ time and only has an effect on that moment, for example, the initial price subsidy for electric energy substitution is not likely to last for a long time, and the intervention variable can be expressed by a pulse function:

$$
P_{t}^{(T)}=\left\{\begin{array}{l}
0, t \neq T \\
1, t \geq T
\end{array}\right.
$$

The intervention variable is the basic element in the intervention analysis model. It can only indicate the time of the intervention and can't completely reflect the influence degree and the gradual influence process of the intervention. Sometimes after the intervention, the original sequence is not immediately affected, but there is a certain lag period. Sometimes the original sequence may gradually rise or fall, and may return to its original level or reach a new level after intervention. We need to deal with the intervention variables in the form of intervention events.

\section{B. Forms of Policy Intervention Events}

The existing policies and the possible new policies in the future are divided into two categories according to the time dimension, which are short-term policies and medium and long term policies respectively. Short-term policies mainly consider prices, subsidies and environmental policies. The medium and long term policy mainly considers the regional planning, the industrial planning and the equipment environmental protection standard policy.

Aiming at the characteristics of various electric energy substitution policies, a simulation model of policy intervention is set up, and the related policy simulation calculation is carried out. Include electric energy substitution short-term policy simulation based on optimal subsidy allocation, renewable energy generation electricity price, energy saving and emission reduction. Include electrical energy substitution policy simulation in the medium and long term based on regional planning, industrial planning, equipment standards, and environmental standards. Include electric energy substitution cost social sharing mechanism choice based on multi-regional and multi-industry.

Based on the policy characteristics of various types of electrical energy substitution, according to the impact mode and duration of the policy impact intervention, the electric energy substitution policy impact events can be summarized into the following four types. As shown in Table 1 below:

TABLE I. TYPES OF INTERVENTION EVENTS

\begin{tabular}{|c|c|c|}
\hline & $\begin{array}{c}\text { Policy impact lasted } \\
\text { for a long time }\end{array}$ & $\begin{array}{c}\text { Policy impact lasted } \\
\text { for a short time }\end{array}$ \\
\hline $\begin{array}{c}\text { Policy impact } \\
\text { unchanged }\end{array}$ & a) & c) \\
\hline $\begin{array}{c}\text { Policy impact } \\
\text { changed gradually }\end{array}$ & b) & d) \\
\hline
\end{tabular}

The intervention models corresponding to the four types of electrical energy substitution policy impact events are as follows:

a) The electric energy substitution policy affects the event to begin relatively quickly, and the influence of the intervention lasts for a long time. Assuming that the policy has an effect on the dependent variable starting from a certain point $\mathrm{T}$, the degree of influence is fixed and unknown. w indicates the intensity of the effect. For the unknown permanent change of the mean caused by the intervention, it can indicate the change 
of the mean of the energy substitution amount caused by the energy substitution policy. The intervention model is:

$$
Y_{t}=\omega S_{t}^{(T)}
$$

If the intervention occurs at $t$ time, but the effect begins to appear after the delay of the $b$ time unit. That is, at $t+b$ time, the intervention model can be adjusted to:

$$
Y_{t}=\omega S_{t+b}^{(T)}=\omega B^{b} S_{t}^{(T)}
$$

Such models can be used to represent and simulate long-term industry regulations implemented at a given moment. In the electric energy substitution policy, the model can be used to simulate and analyze the industry standard implemented by each electric energy substitution industry.

b) The effect of power substitution policy is gradually beginning, and the influence of intervention lasts for a long time. In practice, interventions do not have a full impact immediately at the beginning, and their full impact will take a long time to be fully realized. The simplest intervention model in this form is:

$$
Y_{t}=\frac{\omega B}{1-\delta B} S_{t}^{(T)}, 0<\delta<1
$$

If the intervention occurs at $t$ time, but the effect begins to appear after the delay of the $b$ time unit. That is, at $t+b$ time, the intervention model can be adjusted to:

$$
Y_{t}=\frac{\omega B^{b}}{1-\delta B} S_{t}^{(T)}, 0<\delta<1
$$

Such models can be used to represent and simulate long-term planning that starts at a given moment. In the power substitution policy, the model can be used to simulate and analyze the regional planning and industry planning of each electric energy substitution industry. Because long-term regional and industry planning begins at some point, the degree of impact is a gradual state. Long-term planning does not have an immediate effect in the early stages and will impact gradually over time.

c) The effects of the intervention occur suddenly and for a short period of time. For example, the impact of energy substitution policy on sudden events, such intervention effects can be expressed by impulsive virtual variables. The intervention model is:

$$
Y_{t}=\omega P_{t}^{(T)}
$$

If the intervention occurs at $t$ time, but the effect begins to appear after the delay of the $b$ time unit. That is, at $t+b$ time, the intervention model can be adjusted to:

$$
Y_{t}=\omega P_{t-b}^{(T)}=\omega B^{b} P_{t}^{(T)}
$$

Such models can be used to represent and simulate short-term policies that start at a certain time in a longer sequence of time, with relatively short policy maintenance. In the electric energy substitution policy, the short-term financial and tax subsidies for each electric energy substitution policy can be simulated and analyzed by using this model. For policies such as fiscal subsidies tend to be short-lived after they start to be implemented at some point, produce the characteristics of impulse variables.

d) The effects of intervention events occur suddenly and then gradually weaken to disappear. The model of such intervention events is:

$$
Y_{t}=\frac{\omega B}{1-\delta B} P_{t}^{(T)}, 0<\delta<1
$$

Such models can be used to represent and simulate short-and medium-term policies that start at some point in a longer sequence of time. The model can be used to simulate and analyze the price subsidy policy which keeps the substitution of electric energy for several years in the policy of electric energy substitution. In the power substitution policy, the model can be used to simulate and analyze the price subsidy policy which maintained for several years and decreased the cost gradually. Price subsidy policies tend to last longer in the initial period of subsidies, but then subsidies are gradually reduced until the industry steadily eliminates subsidies.

In the more complicated case of multiple policy parallelism, the above four cases can be used in combination, that is, multiple policy parallelism. The intervention model of electric power substitute policy is to add various interfering variables into the time series of energy substitution index. According to TTSIM, the smoothed time series mentioned earlier satisfies the following model:

$$
Y_{t}=N_{t}=\frac{\theta_{q}(B) \Theta_{Q}\left(B^{s}\right)}{\nabla^{d} \nabla_{s}^{D} \phi_{p}(B) \Phi_{P}\left(B^{s}\right)} \varepsilon_{t}
$$

Suppose the impact of a policy intervention event is:

$$
m_{t}=\frac{\omega(B)}{\delta(B)} I_{t}^{(T)}
$$

Where $I_{t}^{(T)}$ is intervention variable, which equals to $S_{t}^{(T)}$ or $P_{t}^{(T)}$. The time series intervention model is:

$$
Y_{t}=m_{t}+N_{t}=\frac{\omega(B)}{\delta(B)} I_{t}^{(T)}+\frac{\theta_{q}(B) \Theta_{Q}\left(B^{s}\right)}{\nabla^{d} \nabla_{s}^{D} \phi_{p}(B) \Phi_{P}\left(B^{s}\right)} \varepsilon_{t}
$$

The parameters to be evaluated in the policy intervention model include intervention model parameters, such as $w, \delta$ and $b$. Parameters of time series model before intervention in $\operatorname{ARIMA}(p, d, q) \times(P, D, Q)_{S}$ model are $p, d, q, P, D, Q$ and $S$. 


\section{The Modeling Process of Power Substitution POLICY INTERVENTION}

For the electric energy substitution policy time series intervention analysis model, the following steps can be followed:

a) Observe the time series before and after the promotion of electric energy substitution policy. Determine the nature and type of power substitution policy intervention event preliminarily according to the characteristics of electric energy substitution policy which interferences the time and influence period of electric energy substitution.

b) A time series model is established by using the Energy Substitution time series data. The obtained model is used for extrapolation prediction and the predicted values are taken as the values unaffected by the intervention. That is, assuming that for the implementation of electric energy substitution policy, the normal development according to the law of the value.

c) The actual value of energy substitution after the implementation of the power substitution policy minus the predictive value unaffected by the intervention, and the concrete results of the effect of the electric energy substitution policy on the amount of electric energy substitution are obtained.

d) Electric energy substitution after the implementation of electric energy substitution policy is calculated by using the estimated energy substitution policy intervention model to fit the influence value during the intervention and substitute the actual value with the electrical energy substitution during the intervention minus the fitted value of the intervention model. The real sequence of the energy substitution plus the energy substitution is worth the energy substitution sequence value which is not affected by the energy substitution policy for the whole time period.

e) A time series model was established by using the purification sequence, and the estimated power substitution policy intervention model was used to obtain the total electric energy substitution time series intervention analysis model.

\section{CONCLUSIONS}

This paper studies the key technologies of electric energy substitution policy simulating. By using the technology of policy simulation, we can calculate the influence of electric energy substitution policy on electric power substitution, electrification degree, primary energy structure, economic development, ecological environment and people's life. The simulation results of electric energy substitution policy play an important role in the formulation of electric energy substitution policy, which provides an important supporting role for policy decision makers in policy analysis, comparison and decision making. At the same time, it also provides an important support for enterprises to target support policies to the government ensuring the efficient and effective promotion of electric energy substitution.

\section{ACKNOWLEDGMENT}

This research was supported by the 2017 Science \& Technology Project of State Grid Corporation of China (Research on electric energy substitution planning models, policy simulations and their applications, No. 52130N17000R), the 2018 Self-study Program of State Grid Energy Research Institute Co., Ltd. (Study on short-term policy simulation of electric energy substitution considering electricity price of renewable energy generation, No. 52670017000R), and the Young Talents Programme of State Grid Energy Research Institute Co., Ltd. (Research on the key technology of fog computing for smart grid, No. XM2018020035180).

\section{REFERENCES}

[1] W. Tang, Y. Zhang, F. Luan, Study on Method of Determine the Value of Multi-field Energy Saving Project Promotion [C]. The IET 7th International Conference on Wireless, Mobile \& Multimedia Networks, 2017.

[2] Li Ming, Hu Diangang, Zhou Youxue. Research and practice of renewable energy local consumption mode in Gansu province based on “double alternative”strategy[J]. Power System Technology, 2016, 40(10): 2991-2997.

[3] W. Tang, Scientific Implementation of Electric Energy Substitution Planning [J]. China Power Enterprise Management, 2017.7, pp. 54-55.

[4] Y. Zhang, B. G. Shan, P. Wu, W. Tang, F. Lin, K. Xiong, A Three-Level Sustainability Evaluation Framework for Electric Energy Substitution Project [C]. 2018 International Conference on Energy, Power and Materials Engineering (EPME2018), 2018.

[5] Zhang Jing, Zhang Jianzhong, Marketing strategies and implementation of electric power alternatives[J]. Power Demand Side Management, 2015, 17(1): 41-44.

[6] Peng WU, Yu ZHANG and Mo SHI, The study of the clean heating ways in North China and State Grid Corporation of China's strategy [C]. 2018 International Conference on Energy, Environment and Power Engineering (EEPE2018), 2018

[7] Yu Zhang, Fen Lin, Fengkui Luan and Ke Xiong, Analysis of Electric Energy Substitution Potential Based on Re-electrification Target [C]. 2018 International Conference on Electrical, Control and Mechanical Engineering (ECME2018), 2018.

[8] Lee S, Wang S, Bain P A, et al. Modeling and Analysis of Postdischarge Intervention Process to Reduce COPD Readmissions[J]. IEEE Transactions on Automation Science \& Engineering, 2018, PP(99):1-14. 